\title{
Elaboración de un néctar de granadilla (Passiflora ligularis L.) bajo en calorías
}

\section{Elaboration of low calories pomegranate (Passiflora ligularis L.) nectar}

\author{
Miriam Ramos ${ }^{1, a, *}$, Noemí Bravo ${ }^{2, b}$, Lehninger Obregón ${ }^{1, c}$, Gregorio Cisneros ${ }^{3, d}$ \\ ${ }^{1}$ Escuela Profesional de Ingeniería Agroindustrial, Facultad de Ciencias Agrarias, Universidad Nacional Hermilio \\ Valdizán, Huánuco, Perú. \\ ${ }^{2}$ Escuela Profesional de Ingeniería Agroindustrial, Facultad de Química e Ingeniería Química, Universidad Nacional \\ Mayor de San Marcos, Lima, Perú. \\ ${ }^{3}$ Escuela Profesional de Administración de Negocios Internacionales, Universidad Nacional Intercultural de la Selva \\ Central "Juan Santos Atahualpa", Junín, Perú.
}

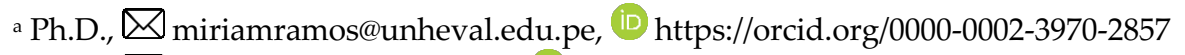

b M.Sc., \nbravoa@unmsm.edu.pe, (D) https://orcid.org/0000-0003-3454-2601

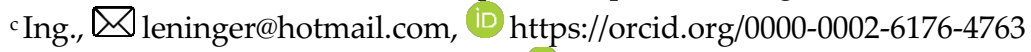

dMg., \ gcisneros@uniscjsa.edu.pe, (ID https://orcid.org/0000-0003-1968-5786

* Autor de Correspondencia: Tel. +51 958578152

http://dx.doi.org/10.25127/riagrop.20213.703

http://revistas.untrm.edu.pe/index.php/RIAGROP revista.riagrop@untrm.edu.pe

Recepción: 04 de junio 2021

Aprobación: 08 de junio 2021

Este trabajo tiene licencia de Creative Commons. Attribution-NonCommercial-ShareAlike $\quad 4.0$ International Public License - CC-BY-NC-SA 4.0

\section{Resumen}

Se formuló un néctar de granadilla bajo en calorías a partir de tres diluciones: D1 (1 zumo: 2.5 agua), D2 (1 zumo: 3 agua) y D3 (1 zumo: 3.5 agua) revelando que el mejor tratamiento correspondió a la dilución 2, mediante una prueba de preferencia ampliada. En la etapa de pasteurización, se evaluaron cuatro formulaciones sustituyendo el $80 \%$ de azúcar: F1 (Azúcar, Acesulfame K $15 \%$, Aspartame $35 \%$, Sucralosa $50 \%$ ), F2 (Azúcar, ED40001), F3 (Azúcar, Acesulfame K $15 \%$, Aspartame $35 \%$ y Estevia 50 \%) y F4 (Azúcar, Estevia 50 \% y Sucralosa 50 \%) hasta obtener el producto final. La prueba de preferencia ampliada evidenció diferencias significativas, seleccionando a tres tratamientos F1, F3 y F4 para continuar con la investigación y evaluar la estabilidad del producto a condiciones ambientales cada 30 días durante 02 meses, que mostraron diferencias significativas. En la evaluación sensorial a través de la prueba no paramétrica de Friedman, se registró como mejor tratamiento a la 
formulación F3, obteniendo un calificativo de "Bueno" en los atributos de sabor (4.08), color (4.33) y aspecto general (4.08), la que se contrastó con la prueba de preferencia ampliada. El mejor tratamiento reportó una composición fisicoquímica de $25.38 \mathrm{Kcal}$ con una reducción de 48.20 \% Kcal respecto al néctar convencional (49 Kcal), sólidos solubles de $6.4{ }^{\circ} \mathrm{Brix}, \mathrm{pH}$ 4.03, acidez $0.34 \%$ expresado en ácido cítrico y $1.73 \mathrm{mg} / 100 \mathrm{~g}$ de vitamina C.

Palabras Clave: Zumo, estabilidad, características sensoriales y bajo en calorías.

\begin{abstract}
A low-calorie pomegranate nectar was formulated from three dilutions: D1 (1 juice: 2.5 water), D2 (1 juice: 3 water) and D3 (1 juice: 3.5 water). The extended preference test revealed that the best treatment was dilution D2. Four formulations were evaluated later during pasteurization, substituting sugar at 80\%: F1 (sugar, 15\% Acesulfame K, 35\% aspartame, 50\% sucralose), F2 (sugar, ED400-01), F3 (sugar, 15\% Acesulfame K, 35\% aspartame, 50\% stevia) and F4 (sugar, 50\% stevia and 50\% sucralose) to obtain the final product. The extended preference test found significant differences for F1, F3 and F4 treatments. From this result the investigation was continued for evaluating the stability of the product at room conditions monthly for 60 days, where significant differences were found. In the sensory assessment by non-parametric Friedman test, the best treatment was the F3 formulation obtaining a good score for taste (4.08), color (4.33) and general aspect (4.08) attributes. This result was contrasted with the extended preference test. The best treatment show a physicochemical composition of $25.38 \mathrm{Kcal}$ with $48.20 \% \mathrm{Kcal}$ reduction in relation to traditional nectar (49 Kcal), $6.4{ }^{\circ}$ Brix soluble solids; $4.03 \mathrm{pH}, 0.34 \%$ acidity expressed in citric acid and $1.73 \mathrm{mg} / 100 \mathrm{~g}$ of vitamin C.
\end{abstract}

Keywords: Zumo, stability, sensory traits, low calories.

\section{INTRODUCCIÓN}

Las bebidas azucaradas dominan el mercado peruano y traen consecuencias en la salud de los consumidores de diferentes edades. Se propone el diseño de un néctar de granadilla bajo en calorías dirigido a la prevención de enfermedades como la diabetes mellitus tipo II, obesidad y enfermedades cardiovasculares, de tal modo que se obtenga un producto estable en el tiempo y que represente una alternativa en el mercado. Actualmente, existen edulcorantes no nutritivos dentro los cuales se encuentra la sucralosa, aspartamo, Acesulfame K y Stevia, como naturales que son edulcorantes aprobado para su uso. Para cada edulcorante, la FDA y la EFSA indican una IDA, que es la cantidad de edulcorante que se considera segura para consumir a diario (Schiano et al., 2021). En este sentido, se presentan alternativas de transformación, donde el fruto de la granadilla (Passiflora ligularis L.) es utilizado para preparar jugos, mermeladas, bebidas fermentadas $y$ néctares que la hacen atractiva por sus propiedades medicinales (hipoalérgico $y$ laxativo) debido a la presencia de fibra. Su cultivo se desarrolla en los valles interandinos como la sierra, selva alta y costa. Anualmente en la región Huánuco, Perú, se produce 3500 toneladas de granadilla, en 700 hectáreas ubicadas en los distritos de Churubamba y Chinchao (Huánuco); Chaglla, Umari y Molino (Pachitea) y Conchamarca (Ambo) (Basilio, 2012). 
Se planteó como objetivos la caracterización de la granadilla, formular un néctar y estudiar la estabilidad durante el almacenamiento.

\section{MATERIALES Y MÉTODOS}

El trabajo de investigación se realizó en las instalaciones del Centro de investigación y transferencia tecnológica agroindustrial (CITTA), laboratorio de Bromatología de la Escuela Profesional de Ingeniería Agroindustrial de la Universidad Nacional Hermilio Valdizán, Huánuco, Perú. El procesamiento del néctar bajo en calorías fue a nivel experimental con los materiales y métodos siguientes:

\subsection{Materia prima, insumos, envases $y$ reactivos}

Como materia prima se usó granadilla ecotipo criollo mejorado, procedente de Tambillo, distrito de Umari, provincia de Pachitea y Región de Huánuco. Además, se usó azúcar blanca, edulcorantes no nutritivos (Estevia, sucralosa, aspartame, Acesulfame K y Edulcor ED400-01), ácido cítrico, carboximetilcelulosa sódica (CMC) y envases de vidrio de $250 \mathrm{ml}$.

Como reactivos se empleó Hidróxido de sodio $(\mathrm{NaOH})$, fenoftaleína, ácido ascórbico, ácido oxálico, colorante (2-6 Diclorofenol indofenol), ácido nitro salicílico (DNS) y otros reactivos especificados en los métodos de análisis.

\subsection{Análisis físico-químico}

2.2.1. Características biométricas de la granadilla: Se registró la longitud, diámetro y peso (Ramos, 2011a).

2.2.2. Humedad, materia seca, proteína, $p H$ y sólidos solubles: Por el método A.O.A.C (1997).
2.2.3. Acidez: Basada en la técnica volumétrica, con resultados expresados en el porcentaje de ácido cítrico (Pearson, 1976).

2.2.4. Vitamina C: Se determinó cuantitativamente por el método espectrofotométrico, basado en la reducción del colorante 2,6 diclorofenol indofenol por una solución de ácido (Ramos, 2010).

2.2.5. Azúcares reductores: Se determinó según lo establecido por Miller (1959), citado por Ramos (2011a).

\subsection{Evaluación sensorial}

La evaluación sensorial se realizó con un panel conformado por 15 jueces semientrenados, quienes efectuaron las evaluaciones para encontrar la mejor dilución de zumo/agua mediante la prueba de preferencia ampliada.

Para la selección del mejor edulcorante, se realizó la prueba de preferencia ampliada y, para los atributos de calidad más relevantes de este producto, se realizó la prueba de aceptabilidad utilizando la Escala de Likert. Los datos fueron tratados estadísticamente mediante la prueba no paramétrica de Friedman $(\alpha=0,05)$ (Montgomery, 2004 \& Sotomayor, 2008).

\subsection{Metodología experimental}

2.4.1. Estudio de las formulaciones del néctar de granadilla bajo en calorías: Para la elaboración de este producto se empleó el flujo de procesamiento mostrado en la figura 1.

Las operaciones más importantes fueron la estandarización, que permitió encontrar la 
mejor dilución del zumo: agua, y el estudio de las formulaciones del néctar, que consistió en sustituir el azúcar por los edulcorantes y se tuvo en consideración la normativa internacional para los productos bajos en calorías. Después, mediante evaluaciones sensoriales sucesivas, se encontró a los tratamientos más destacados que permitieron continuar con el estudio y evaluar su estabilidad durante el almacenamiento.

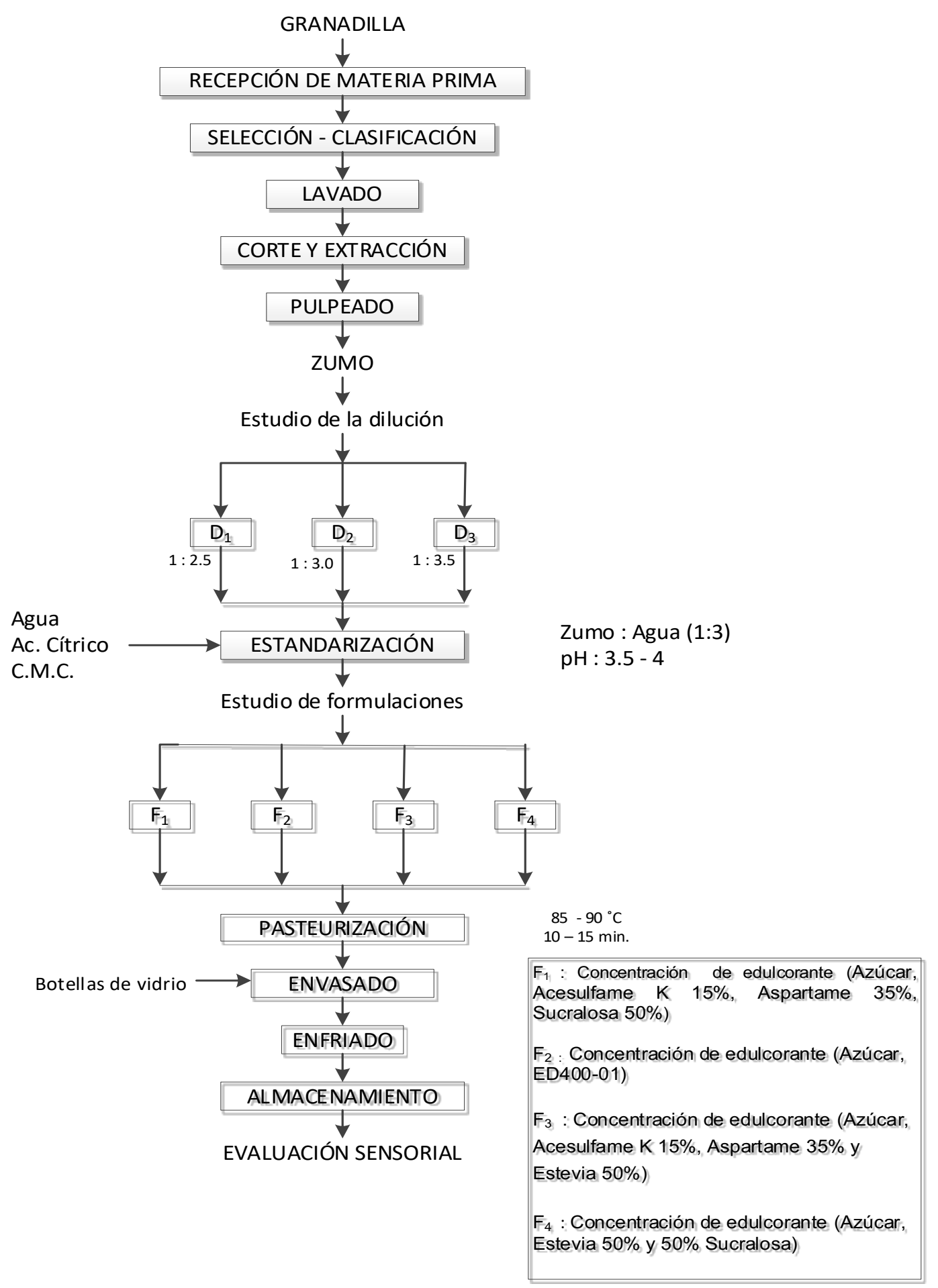

Figura 1. Diagrama de bloques del proceso de elaboración del néctar de la granadilla bajo en calorías. 
2.4.2. Estudio de las formulaciones durante el almacenamiento: De acuerdo con los resultados de la evaluación sensorial, mediante la prueba de preferencia ampliada, se seleccionó tres tratamientos (F1, F3 y F4), a fin de evaluar la estabilidad en el almacenamiento e identificar al mejor tratamiento a través de la prueba de preferencia y prueba de aceptabilidad a fin de caracterizarlo.

\subsubsection{Caracterización del néctar de granadilla bajo} en calorías: Los valores obtenidos del análisis estadístico y sensorial fueron tabulados y evaluados estadísticamente. El mejor tratamiento fue caracterizado según análisis especificados en los métodos propuestos.

\subsection{Análisis estadístico}

Los resultados de $\mathrm{pH}$, sólidos solubles y acidez (por triplicado) del néctar bajo en calorías, almacenados a condiciones ambientales, fueron monitoreados cada 30 días durante 02 meses. Los datos obtenidos fueron procesados estadísticamente a fin de seleccionar al mejor tratamiento. Se utilizó un diseño completamente al azar (DCA) y prueba de Tukey, con un nivel de significación de 0,05 \% y se utilizó el paquete estadístico Statgraphics Plus versión 5.1 .

\section{RESULTADOS Y DISCUSIÓN}

\subsection{Caracterización de la materia prima}

La tabla 1 muestra los resultados de los análisis físico-químicos de la granadilla.
Tabla 1. Resultados de los análisis fisicoquímicos de la granadilla

\begin{tabular}{lc}
\hline Características & $\begin{array}{c}\text { Resultados } \\
(\%)\end{array}$ \\
\hline Humedad (\%) & 83.73 \\
Materia seca (\%) & 16.27 \\
Sólidos solubles ( ${ }^{\circ}$ Brix) & 15.20 \\
$\mathrm{pH}$ & $4.40-4.86$ \\
Acidez (\% expresado en ácido & 0.64 \\
cítrico) & \\
Color & \\
$\mathrm{L}^{*}$ & 63.22 \\
$\mathrm{a}^{*}$ & 21.33 \\
$\mathrm{~b}^{*}$ & 52.33 \\
Azúcares reductores (mg/100g) & 7.99 \\
Vitamina C (mg/100g) & 15.99 \\
Longitud (mm) & 65.20 \\
Diámetro ecuatorial (mm) & 58.42 \\
Rendimiento de zumo (\%) & 15.89 \\
\hline
\end{tabular}

\subsection{Estudio de las formulaciones del néctar bajo en calorías}

Los resultados obtenidos para establecer la dilución óptima (zumo: agua) fueron sometidos a una evaluación sensorial que, posteriormente con la mejor dilución, permitieron diseñar cuatro formulaciones con diversas mezclas de edulcorantes planteadas en la figura 1. Estas fueron llevadas a un análisis sensorial a través de la prueba de preferencia ampliada, con 15 panelistas semientrenados con dos repeticiones (ver tabla 2) para poder identificar los tratamientos más destacados.

Tabla 2. Resultados de significancia de la evaluación sensorial

\begin{tabular}{ccc}
\hline Tratamientos & R1 & R2 \\
\hline F3 y F1 & n.s & $*$ \\
F3 y F4 & $*$ & $*$ \\
F3 y F2 & $*$ & $*$ \\
F1 y F4 & n.s & n.s \\
F1 y F2 & $*$ & $*$ \\
F4 y F2 & $*$ & $*$ \\
\hline n.s. : No existe diferencia significativa entre tratamientos \\
: Existe diferencia significativa entre tratamientos
\end{tabular}




\subsection{Estudio de las formulaciones durante el} almacenamiento

En las figuras 2, 3 y 4 se puede observar que los sólidos solubles, $\mathrm{pH}$ y acidez en las formulaciones F1, F3 y F4 de estas bebidas, durante el almacenamiento a condiciones ambientales, mostraron ligeras variaciones con el tiempo. En la evaluación estadística se reportó diferencias significativas a un nivel de significancia al $5 \%$.

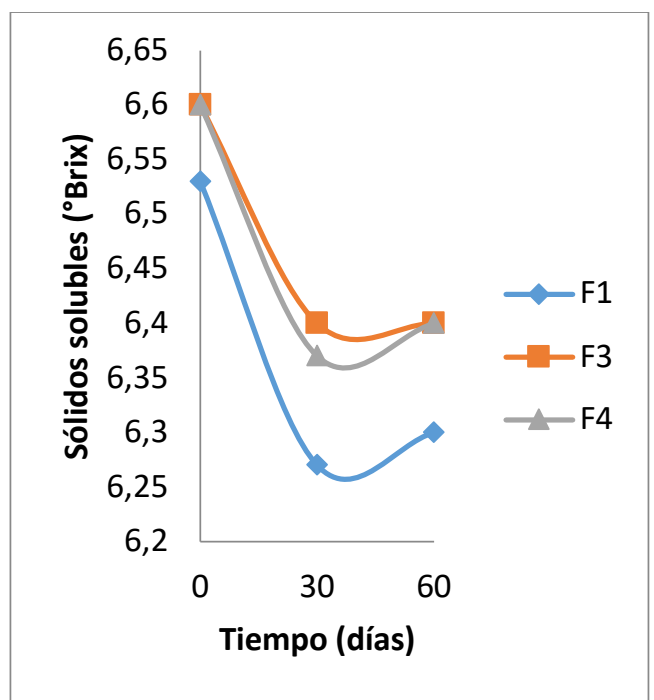

Figura 2. Variación de sólidos solubles durante el almacenamiento.

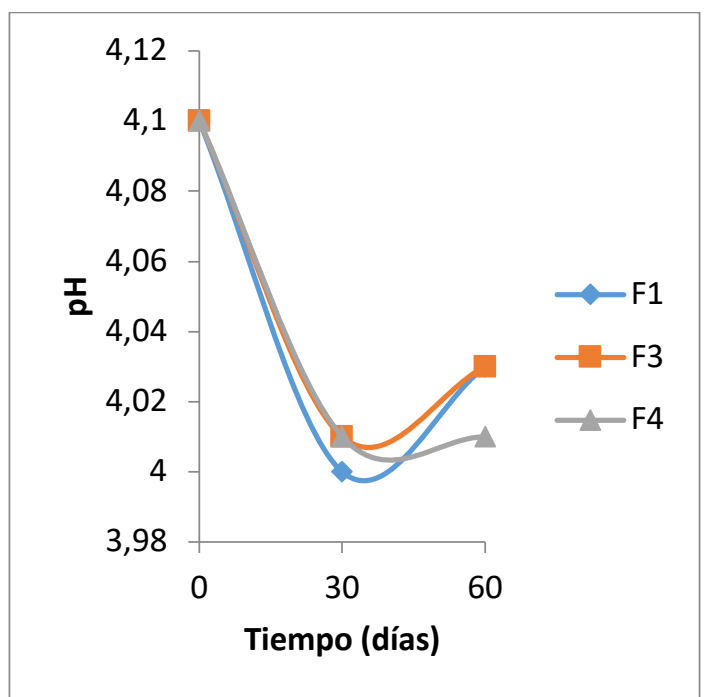

Figura 3. Variación de $\mathrm{pH}$ durante el almacenamiento.

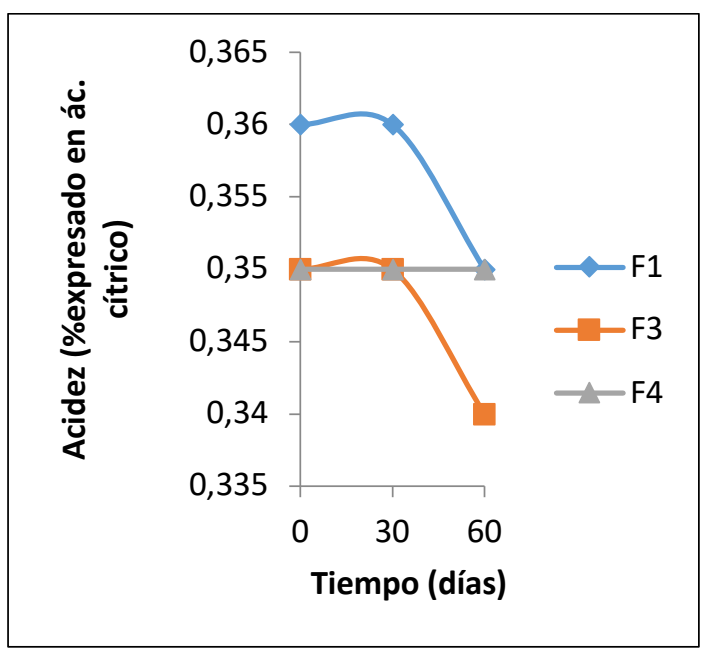

Figura 4. Variación de acidez (\% expresado en ácido cítrico) durante el almacenamiento.

\subsection{Evaluación sensorial}

En la tabla 3, se registra los resultados de la evaluación sensorial que se efectuó a los 60 días de almacenamiento, mediante un panel entrenado de 15 personas quienes evaluaron los atributos de sabor, color y aspecto general.

Tabla 3. Resultados de la evaluación sensorial

\begin{tabular}{crrc}
\hline Tratamientos & Sabor & Color & $\begin{array}{c}\text { Aspecto } \\
\text { General }\end{array}$ \\
\hline F4 & $3.58^{\mathrm{a}}$ & $3.92^{\mathrm{a}}$ & $4.17^{\mathrm{a}}$ \\
F1 & $3.41^{\mathrm{a}}$ & $4.08^{\mathrm{a}}$ & $3.75^{\mathrm{a}}$ \\
F3 & $4.08^{\mathrm{a}}$ & $4.33^{\mathrm{a}}$ & $4.08^{\mathrm{a}}$ \\
\hline
\end{tabular}

\subsection{Caracterización del néctar de granadilla bajo en calorías}

La tabla 4 muestra la composición fisicoquímica de los tratamientos (F1, F3 y F4) bajos en calorías. 
Tabla 4. Resultados del análisis fisicoquímico

\begin{tabular}{lrrr}
\hline Característica & \multicolumn{1}{c}{ F1 } & \multicolumn{1}{c}{ F3 } & \multicolumn{1}{c}{ F4 } \\
\hline Humedad g/100g de muestra & 93.65 & 93.66 & 93.43 \\
Materia seca & 6.35 & 6.34 & 6.57 \\
Proteína cruda g/100g de muestra & 0.15 & 0.15 & 0.13 \\
Carbohidratos g/100g & 6.20 & 6.19 & 6.44 \\
Energía Total Kcal/100g & 25.40 & 25.38 & 26.28 \\
pH & 4.03 & 4.03 & 4.01 \\
Sólidos solubles ( ${ }^{\circ}$ Brix) & 6.30 & 6.40 & 6.40 \\
Acidez (\%expresado en ac. Cítrico) & 0.35 & 0.34 & 0.35 \\
Vitamina C (mg/100g muestra) & - & 1.73 & - \\
\hline
\end{tabular}

De acuerdo a la tabla 1, la humedad en la granadilla reportó $83.73 \%$, equivalente en materia seca a $16.27 \%$, valores cercanos a los estudios de Cabrera (2006) y Malca (2001). Las variaciones, probablemente, difieran por las prácticas agrícolas, suelo, condiciones climatológicas entre otros factores y este porcentaje se ubica dentro de la composición de frutas y hortalizas frescas (Ramos, 2011a).

Los sólidos solubles arrojaron $15.2{ }^{\circ}$ Brix, que son valores que se ubican en el marco de los estudios realizados por Cabrera (2006) e INCOTEC (1997).

El pH fue 4.40-4.86, valores por debajo a los encontrados por Cabrera (2006), pero que se ubican dentro del rango de 4.3 a 4.6 característicos de frutos que alcanzaron su madurez comercial (Orjuela et al., 2002 \& Márquez et al., 2009).

Referente a la acidez, expresada en ácido cítrico, se reveló $0.64 \%$, valores cercanos a los reportados por Cabrera (2006) y con diferencias a los registrados, que probablemente se deban a los aspectos comentados anteriormente (Orjuela et al., 2002).

El color instrumental en las coordenadas CIELAB reportaron $L^{*}=63.22, \quad a^{*}=21.33 \quad y$ $b^{*}=52.33$ y fueron los valores de $b^{*}$ característicos de la coloración amarilla propia de esta fruta, que puede variar de acuerdo a su estado de madurez (Ramos, 2011a).

El contenido de azúcares reductores registró $7.99 \mathrm{mg} / 100 \mathrm{~g}$, datos cercanos a los publicados por Cabrera (2006). El zumo de esta fruta es rico en azúcares (sacarosa, glucosa y fructosa), que aumenta como producto de la hidrólisis del almidón a medida que madura (Orjuela et al., 2002).

La vitamina C reveló un contenido de 15.99 $\mathrm{mg} / 100 \mathrm{~g}$, semejante a los estudios propuestos por Cabrera (2006), pero que difieren, probablemente, por las prácticas agronómicas, suelo, condiciones climatológicas entre otros factores propios de cada país (Cabrera, 2006 \& Cerdas y Castro, 2003).

Respecto a las características biométricas, la longitud fue $65.20 \mathrm{~mm}$ y diámetro ecuatorial $58.42 \mathrm{~mm}$, relacionadas geométricamente a la forma redonda. Estos datos fueron cercanos a los valores encontrados en longitud $(7.24 \mathrm{~cm}) \mathrm{y}$ diámetro $(6.85 \mathrm{~cm})$, que cambia de acuerdo con la variedad (Villamizar y Gutiérrez, 2009).

El rendimiento en zumo fue $15.89 \%$ y se encontró un bajo rendimiento en la parte comestible (endocarpio) respecto al peso total. Este detalle se debe tener en cuenta en su comercialización, cuyos deshechos pueden ser muy bien aprovechado en la alimentación animal. Al respecto, se obtuvo rendimientos de las partes comestibles (endocarpio-semilla) de 30.00 a $60.55 \%$ para los ecotipos común y colombiano en productores más representativos de la región Huánuco (Ramos, 2011b), resultados que difieren a los encontrados en razón a registrarse el rendimiento respecto al endocarpio.

Del estudio de dilución, se reveló a través de una prueba de preferencia ampliada que el 
mejor tratamiento corresponde a la dilución (1 zumo de granadilla: 3 Agua), asociado con el menor puntaje, equivalente a una mayor preferencia.

Según la tabla 2, del estudio de formulaciones con la utilización de los edulcorantes señalados en la figura 1, la prueba de comparaciones múltiples por Tukey muestra, tanto en la primera y segunda réplica, diferencias significativas a un $5 \%$ en todas las formulaciones a excepción de las formulaciones F3 y F1 con F1 y F4 que no presentan diferencias significativas. De esta evaluación, se puede concluir que las muestras de mayor preferencia fueron las que reportaron menores puntajes, seleccionando a las formulaciones F1, F3 y F4 para continuar con el estudio.

De las Figuras 2, 3 y 4, los atributos de calidad se encontraron dentro del rango permitido (INACAL, 2009) y los productos bajos en calorías más comercializados (Ramos y Obregón, 2012).

Referente a la evaluación sensorial, los atributos más relevantes de este producto no reportaron diferencias significativas entre tratamientos. Sin embargo, a través de la escala de Likert, la formulación F3 presentó el mejor puntaje en los atributos sabor (4.08) y color (4.33), mientras el aspecto general reportó calificativos de $4.17 \mathrm{y}$ 4.08 para las formulaciones F4 y F3, que categorizan al producto con la denominación de bueno (4), según la escala de evaluación utilizada. El análisis estadístico de Friedman ratificó lo antes expuesto, a un nivel de significación de $5 \%$. Al respecto, también este estudio se contrastó con la prueba de preferencia realizada para las tres formulaciones, no encontrando diferencias significativas entre los tratamientos al emplear la prueba de preferencia ampliada. Sin embargo, el menor puntaje fue asociado con la mejor preferencia correspondiente a la formulación F3.

El aporte energético (tabla 4) se realizó en función a los macronutrientes multiplicados por el factor de conversión y se consideró un bajo contenido de grasa, que resultó como la mejor formulación (F3) con un contenido de 25.38 $\mathrm{Kcal} / 100 \mathrm{~g}$ de producto, respecto a las formulaciones F1 (25.40 Kcal) y F4 $(26.28 \mathrm{Kcal})$ comparado con el aporte calórico de un néctar convencional (49 Kcal), que permitió una reducción de $48.20 \%$. Referente a los otros atributos, se muestran semejanzas en razón a que todas las formulaciones partieron de una misma dilución estandarizada y fue la variación en la adición de los edulcorantes no nutritivos. Los valores están dentro de los límites permitidos (INACAL, 2009 \& Ramos y Obregón, 2012). El contenido de vitamina C se registró $1.73 \mathrm{mg} / 100 \mathrm{~g}$, en el mejor tratamiento, donde existió una reducción respecto a la materia prima en razón a la estandarización efectuada al momento de formular el néctar.

\section{CONCLUSIONES}

Se concluye que la granadilla ecotipo criollo mejorado presentó la siguiente caracterización: humedad $83.73 \%$, sólidos solubles $15.2{ }^{\circ}$ Brix, acidez $0.64 \%$ expresado en ácido cítrico, vitamina C $15.99 \mathrm{mg} / 100 \mathrm{~g}$, coordenadas de color $\mathrm{L}^{*}=63.22, \mathrm{a}^{*}=21.33, \mathrm{~b}^{*}=52.33 \mathrm{y}$ rendimiento de zumo de $15.89 \%$. Se logró elaborar un néctar de granadilla bajo en calorías a partir de una estandarización (zumo/agua = 1:3, pH 3.5 a 4) y pasteurización $85-90{ }^{\circ} \mathrm{C}$ por 10 $15 \mathrm{~min}$, mediante la sustitución del $80 \%$ de azúcar por edulcorantes no nutritivos de una mezcla de Acesulfame K 15 \%, Aspartame $35 \%$ 
y Estevia $50 \%$ que calificaron sensorialmente como buena a la mejor formulación F3 y con un valor energético de $25.38 \mathrm{Kcal}$, sólidos solubles de $6.4{ }^{\circ}$ Brix, $\mathrm{pH} 4.03$, acidez $0.34 \%$ expresado en ácido cítrico, y vitamina C $1.73 \mathrm{mg} / 100 \mathrm{~g}$. Se recomienda este producto a las personas que tiene ciertas restricciones en la salud, por su consumo de alimentos elevados en azúcar que son perjudiciales y con perspectivas de su aprovechamiento por sus propiedades digestivas y buen sabor.

\section{AGRADECIMIENTOS}

Nuestro reconocimiento a la empresa Linros Interinsumos S. R. L. en especial al Ing. Juan Manuel Luján por el apoyo con los edulcorantes no nutritivos utilizados en el estudio. Al Ph.D. Oscar Benjamín Jordán, por sus valiosos aportes en la presente investigación. A los Estudiantes de la E.P. de Ingeniería Agroindustrial, Facultad de Ciencias Agrarias, UNHEVAL.

\section{Referencias}

AOAC. 1997. Oficial Methods of Análisis. Agricultura Chemicals, Contaminants; Drugs. Vol I y II. $15^{\text {th }}$ Edición.

Basilio, L. 2012. Huánuco plantea aumentar producción de granadilla para la exportación. Consultado 1 enero 2014. Disponible en: http://elcomercio.pe/economia/ peru/huanuco-plantea-aumentar-producciongranadilla-exportacion-noticia-1443465.

Cabrera, C. 2006. Caracterización de las propiedades físicas y químicas del fruto de Granadilla, Passiflora ligularis Juss. Facultad de Ciencias Agropecuarias y Ambientales. Universidad Técnica del Norte Instituto Ecuatoriano de Normalización.

Cerdas, MdM y Castro, J. 2003. Manual práctico para la producción, cosecha y manejo postcosecha del cultivo de granadilla (Plasiflora ligularis, Juss). Ministerio de Agricultura y Ganadería Consejo Nacional de Producción Universidad de Costa Rica. San José Costa Rica.
Instituto Colombiano de Normas Técnicas y Certificación. Santa Fe de Bogotá: INCOTEC.1997.

INACAL. 2009. Norma Técnica Peruana Jugos, néctares y bebidas de fruta. 203.110. Primera Ediciòn.Lima-Perú. $29 \mathrm{p}$.

Malca, O. 2001. Seminario de Agronegocios Granadilla. Extracto y fresco. Universidad del Pacífico.

Márquez, C. Peláes, M. y Cortes, M. 2009. Deshidratación de granadilla (Passiflora ligularis Juss) por convección forzada para la elaboración de bebidas aromáticas. Revista CES/Medicina Veterinaria y Zooctenía, 4(2).

Montgomery, D. 2004. Diseño y análisis de experimentos. Universidad Estatal de Arizona. Editorial Limusa Wiley.

Orjuela, J.; Pinilla, A. y Rincón, J. 2002. Aplicación de la tecnología de atmósfera controlada para la conservación de la granadilla. Ciencia Investigación Académica Desarrollo.

Pearson, D. 1976. Técnicas de laboratorio para el análisis de los alimentos. Editorial Acribia. Zaragoza. España.

Ramos, M. 2010.Guía de prácticas de control de calidad. E.A.P. Ingeniería Agroindustrial. UNHEVAL, Huánuco.

Ramos, M. 2011a. Efecto del tratamiento hidrotérmico en la conservación postcosecha del tomate (Solanum lycopersicum, L).cv Nabateo. Tesis para Optar el grado de Magister Scientiae en Tecnología de Alimentos. Universidad Nacional Agraria La Molina.

Ramos, M. 2011b. Análisis de la granadilla en VI FestivalHuánuco. Ministerio de Agricultura.

Ramos, M. y Obregón, L. 2012. Análisis fisicoquímico del néctar comercial. Laboratorio de Bromatología UNHEVAL, Huánuco.

Schiano, C., Grimaldi, V., Scognamiglio, M., Costa, D., Soricelli.A. ,Nicoletti, G.f. y Napoli.C. 2021. Soft drinks and sweeteners intake: Possible contribution to the development.of metabolic syndrome and cardiovascular diseases. Beneficial or detrimental action of alternative sweeteners.142

Sotomayor, R. 2008. Métodos estadísticos para la investigación. Departamento de Estadística e informática. Universidad Nacional Agraria La Molina. Lima-Perú.

Villamizar, F. y Gutiérrez. 2009. La granadilla, su caracterización física y comportamiento postcosecha. Revista Ingeniería agrícola. Universidad Nacional de Colombia. 